\title{
The Ultra-Low-k Dielectric Materials for Performance Improvement in Coupled Multilayer Graphene Nanoribbon Interconnects
}

\author{
Peng $\mathrm{Xu}{ }^{1} \oplus$, Zhongliang Pan ${ }^{1, *}$ and Zhenhua Tang ${ }^{2}$ \\ 1 School of Physics and Telecommunication Engineering, South China Normal University, Guangzhou 510006, China \\ 2 School of Electronics Information and Electrics Engineering, Xiangnan University, Chenzhou 422300, China \\ * Correspondence: panzhongliang@m.scnu.edu.cn; Tel.: +86-156-2647-7786
}

Received: 12 July 2019; Accepted: 29 July 2019; Published: 31 July 2019

check for updates

\begin{abstract}
The ultra-low-k dielectric material replacing the conventional $\mathrm{SiO}_{2}$ dielectric medium in coupled multilayer graphene nanoribbon (MLGNR) interconnects is presented. An equivalent distributed transmission line model of coupled MLGNR interconnects is established to derive the analytical expressions of crosstalk delay, transfer gain, and noise output for $7.5 \mathrm{~nm}$ technology node at global level, which take the in-phase and out-of-phase crosstalk into account. The results show that by replacing the $\mathrm{SiO}_{2}$ dielectric mediums with the nanoglass, the maximum reduction of delay time and peak noise voltage are $25.202 \mathrm{~ns}$ and $0.102 \mathrm{~V}$ for an interconnect length of $3000 \mu \mathrm{m}$, respectively. It is demonstrated that the ultra-low-k dielectric materials can significantly reduce delay time and crosstalk noise and increase transfer gain compared with the conventional $\mathrm{SiO}_{2}$ dielectric medium. Moreover, it is found that the coupled MLGNR interconnect under out-of-phase mode has a larger crosstalk delay and a lesser transfer gain than that under in-phase mode, and the peak noise voltage increases with the increase of the coupled MLGNR interconnect length. The results presented in this paper would be useful to aid in the enhancement of performance of on-chip interconnects and provide guidelines for signal characteristic analysis of MLGNR interconnects.
\end{abstract}

Keywords: MLGNR interconnects; crosstalk delay; noise output; transfer gain; ultra-low-k dielectric materials

\section{Introduction}

As the feature size of very large-scale integrated (VLSI) circuits is scaled down to the nanometer order, various performance degradation and stability problems on the conventional $\mathrm{Cu}$ interconnects have emerged in recent years [1-3]. Graphene, as a promising candidate for replacing the common copper, has attracted the intensive interest of many researchers in terms of its excellent electrical, mechanical and thermal properties [4,5]. Compared with the copper material, high quality graphene has a long mean free path on the order of several micrometers, which can result in a lower resistivity and achieving the ballistic transport at shorter interconnects. The current density of graphene can reach $10^{9} \mathrm{~A} / \mathrm{cm}^{2}$ in comparison to its Cu counterpart, thereby, eliminating the electromigration and skin effect of $\mathrm{Cu}$ interconnects [6]. Graphene nanoribbon (GNR) is a narrow strip of graphene sheet, which can be classified into multilayer GNR (MLGNR) and single-layer GNR (SLGNR) depending on its stacked number of layers. SLGNR is not suitable for on-chip interconnects owing to its larger intrinsic resistance [7-9]. Based on the types of connection with other devices or interconnects, MLGNR can be further categorized into top contact MLGNR (TC-MLGNR) and side contact MLGNR (SC-MLGNR) [10-12]. TC-MLGNR has only the top most layer connected to surrounding contacts while all layers of SC-MLGNR are coupled with the other contacts, which results in the distributed 
scattering resistance of the former over the latter. Hence the SC-MLGNR is adopted as interconnect material in this paper.

With the interconnect width shrinking into the nanometer scale, the crosstalk delay, crosstalk noise, and transfer gain of global interconnects in VLSI circuits have become major performance concerns owing to the longer distance. To date, a series of studies about these issues on MLGNR interconnects have been done. In reference [13], Agrawal et al. presented the analytical model of crosstalk delay and crosstalk noise based on the FDTD method. In references [14-16], the propagation delay and transfer gain for a single-line of MLGNR interconnect were investigated. In reference [17], Sahoo et al. analyzed the characteristic of crosstalk noise of coupled MLGNR interconnects, considering the coupling capacitance. In reference [1], Zhao et al. analyzed and compared the performance difference of crosstalk noise and crosstalk delay between coupled MLGNR and $\mathrm{Cu}$ interconnects, considering the impact of coupling capacitance.

However, the works mentioned above only focus on how to establish the analytical model but do not propose the optimization method to reduce crosstalk delay and crosstalk noise and increase transfer gain of on-chip interconnects. Apparently, it is crucial to further enhance the MLGNR interconnects performance at the end of the roadmap. There are some reports that replacing the $\mathrm{SiO}_{2}$ with the ultra-low-k dielectric materials $(\mathrm{k} \leq 2)$ as the dielectric medium can reduce the delay of on-chip interconnects [18-22]. Hence, it is also necessary to investigate the effects of the ultra-low-k dielectric materials on the crosstalk noise and transfer gain of on-chip interconnects. So far, to the best of our knowledge, there is no research to propose the analytical model of coupled MLGNR interconnects, considering the impact of the ultra-low-k dielectric materials. Therefore, in this paper, an analytical model for crosstalk delay, noise output, and frequency response of coupled MLGNR interconnects with different dielectric materials is proposed.

The overview of development about the ultra-low-k dielectric constant materials is shown as follows. The relative dielectric constant of $\mathrm{SiO}_{2}$ as the most common insulator material is usually equal to 3.9 [20]. The low dielectric constant (low-k) $\mathrm{SiCOH}(\mathrm{k}=3.0)$ is regarded as an effective substitute for the conventional $\mathrm{SiO}_{2}$ and has been successfully integrated in some $130 \mathrm{~nm}$ and $90 \mathrm{~nm}$ VLSI circuits [21]. The emergence of the porosity fabrication technology by plasma-enhanced chemical vapor deposition (PECVD) can reduce the relative dielectric constant of insulator material [21]. By applying the fabrication technology, $\mathrm{SiCOH}$ is processed into porous $\mathrm{p}-\mathrm{SiCOH}(\mathrm{k}=2.4)$ that has been applied at IBM's P7 microprocessor [21]. With the continuous advancement of the porosity fabrication technology, the inorganic ultra-low-k dielectrics, such as nanoglass, have the relative dielectric constant of 1.3 [22]. In view of the current research progress of insulator material of VLSI circuits, the nanoglass is the lowest dielectric constant material. Thus, nanoglass was adopted as the ultra-low-k dielectric constant material for analysis of performance of on-chip interconnect compared with the conventional $\mathrm{SiO}_{2}$ in the paper.

\section{Interconnect Model}

A typical geometry of two-line coupled MLGNR interconnects placed above the ground plane and surrounded by a dielectric medium is exhibited in Figure 1, where the common $\mathrm{SiO}_{2}$ dielectric material is replaced by the ultra-low-k dielectric material. The $W, S, T_{g n r}, T_{o x}$, and $\varepsilon_{r}$ are line width, line space, line height, thickness of insulator dielectric, and relative dielectric constant of the ultra-low-k dielectric material, respectively. The total number of layers for MLGNR depends on the line thickness $T_{g n r}$ and can be expressed as $N_{\text {layer }}=1+$ Integer[ $T_{g n r} / \delta$ ] [23]. Herein the operator Integer[.] means that only the integer part is considered and $\delta(=0.34 \mathrm{~nm})$ is the interlayer spacing between successive GNR layers [24]. 


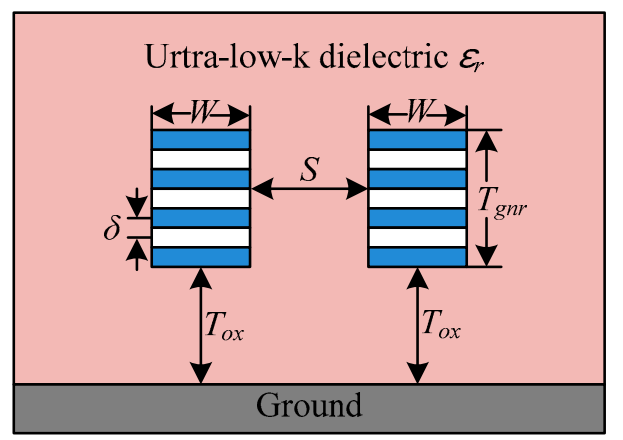

Figure 1. Geometries of two-line coupled multilayer graphene nanoribbon (MLGNR) interconnects using the ultra-low-k dielectric material.

As shown in Figure 2, the equivalent circuit model for two-line coupled MLGNR interconnects is configured with the same effective driver resistance $R_{d}$, driver capacitance $C_{d}$, and load capacitance $C_{l}$ for aggressor and victim lines. The coupled MLGNR interconnects are comprised of lumped and distributed parts.

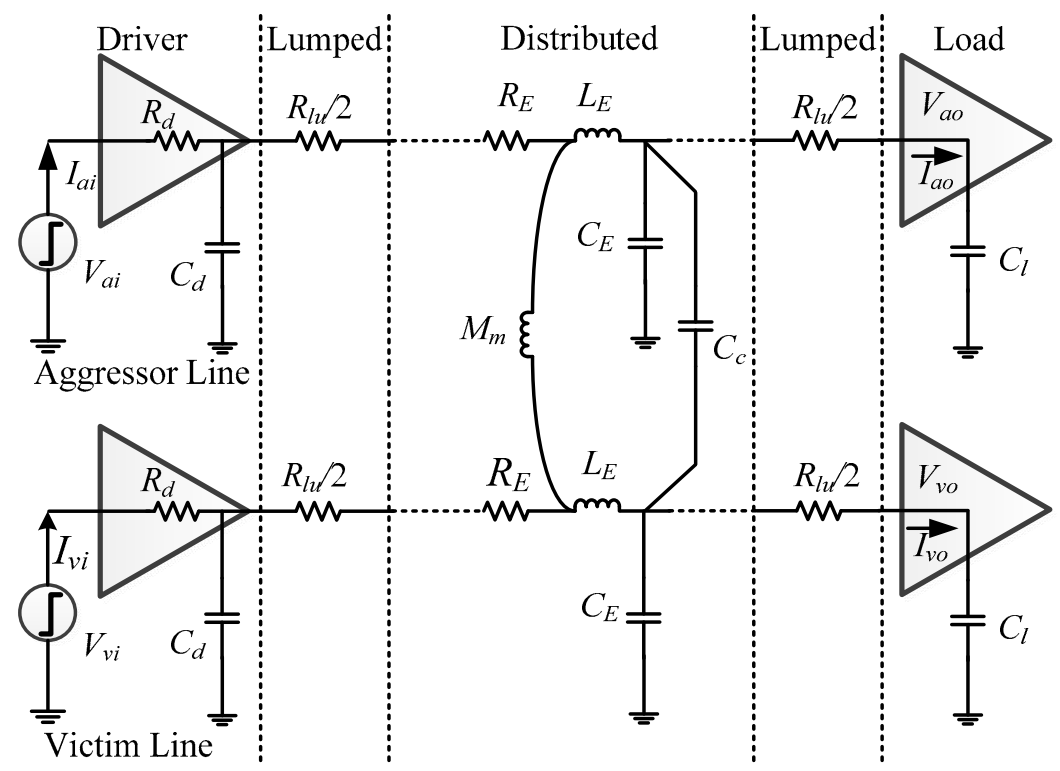

Figure 2. The equivalent circuit model of two-line coupled MLGNR interconnects.

For MLGNR coupled interconnects, $R_{l u}$ and $R_{E}$ represent the lumped resistance and per unit length (p.u.l.) equivalent distributed scattering resistance, respectively. They can be given by [7],

$$
\begin{gathered}
R_{l u}=\frac{R_{c m}+R_{q m}}{N_{\text {layer }} \times N_{c h}}, \\
R_{E}=\left\{\begin{array}{cc}
0 & L_{g n r}<\lambda_{e f f} \\
\frac{R_{q m}}{N_{\text {layer }} \times N_{c h} \times \lambda_{e f f}} & L_{g n r}>\lambda_{e f f}
\end{array} .\right.
\end{gathered}
$$

Here, $R_{c m}$ denotes the imperfect contact resistance and its value ranges from $1 \mathrm{~K} \Omega$ to $20 \mathrm{~K} \Omega$ [13]. $R_{q m}$ is the monolayer quantum resistance and can be defined as $R_{q m}=h / 2 e^{2}$ (herein $h$ is Plank's constant and $e$ is charge of electron). $\lambda_{\text {eff }}$ denotes the effective mean free path (MFP). $N_{c h}$ represents the total number of conducting channels in the monolayer GNR and can be approximated as below $[25,26]$,

$$
N_{c h}=a_{0}+a_{1} W+a_{2} W^{2}+a_{3} E_{f}+a_{4} E_{f} W+a_{5} E_{f}^{2} .
$$


where $a_{0}$ to $a_{5}$ are the parameters for zigzag MLGNR (zz-MLGNR) at room temperature ( $300 \mathrm{~K}$ ) with the Fermi energy $E_{F}$ over 0 [25]. In the light of the total number of conducting channels of zigzag MLGNR over armchair MLGNR (ac-MLGNR), hence, only the zz-MLGNR is investigated in the paper. $L_{g n r}$ represents the length of MLGNR interconnects. The effective mean free path (MFP) for the $i$ th-subband can be expressed as,

$$
\lambda_{e f f, i}=\left(\frac{1}{\lambda_{d}}+\frac{1}{\lambda_{s}}+\frac{1}{\lambda_{e, i}}\right)^{-1}
$$

Herein, $\lambda_{d}$ represents the mean free path due to the scattering effects by the static impurities and crystal defects $(=1 \mu \mathrm{m})[1,10]$. $\lambda_{s}$ denotes the mean free path induced by electron-phonon scattering $(=70 \mu \mathrm{m})$ [27]. $\lambda_{e, i}$ is the mean free path contributed to the scattering of edge roughness, which is depended on the interconnect width and the backscattering probability $P$ at the edges [28].

$$
\lambda_{e, i}=\frac{W}{P} \sqrt{\left(\frac{2 W E_{f}}{i h v_{f}}\right)^{2}-1},
$$

where $v_{f}$ is the Fermi velocity of electrons in graphene $\left(=8 \times 10^{5} \mathrm{~m} / \mathrm{s}\right)$ [12]. The value of $P$ will change when the edge roughness situation is different. Especially, $P=0$ and $P=1$ demonstrate that the edge of MLGNR are fully specular and fully diffusive, respectively [1].

The distributed capacitance $C_{E}$ of MLGNR interconnects comprises of the equivalent quantum capacitance $C_{e q}$ and the electrostatic capacitance $C_{e l}$. The p.u.l. equivalent quantum capacitance can be obtained by using a recursive scheme as $[1,7,17]$,

$$
\begin{gathered}
C_{r e c}^{1}=C_{q}=\frac{4 e^{2} N_{c h}}{h v_{f}}, \\
C_{r e c}^{i}=\left(\frac{1}{C_{r e c}^{i-1}}+\frac{1}{C_{m}}\right)^{-1}+C_{q}, \\
C_{e q}=C_{r e c}^{N_{\text {layer }} .}
\end{gathered}
$$

wherein, $C_{q}$ is the p.u.l. length quantum capacitance of monolayer GNR. $C_{m}$ is the p.u.l. coupling capacitance between successive GNR layers and can be defined as $C_{m}=\varepsilon_{0} W / \delta$ (here $\varepsilon_{0}=8.854 \times 10^{-12}$ is the vacuum dielectric constant). In order to investigate the impacts of ultra-low-k dielectric material materials, thus the relative dielectric constant $\varepsilon_{r}$ can be applied to distinguish different dielectric mediums in this paper. The p.u.l. electrostatic capacitance $C_{e l}$ is determined by the interconnect dimension and relative dielectric constant $\varepsilon_{r}$ of medium material, and can be derived as [29],

$$
C_{e l}=\varepsilon_{r} \varepsilon_{0} M\left[\tanh \left(\frac{\pi W}{4 T_{o x}}\right)\right],
$$

where $M[$.$] can be described as [30],$

$$
M[k]=\left\{\begin{array}{cc}
\frac{2 \pi}{\ln \left(\left(2+2 \times \sqrt[4]{1-k^{2}}\right) /\left(1-\sqrt[4]{1-k^{2}}\right)\right)} & 0 \leq k \leq \frac{1}{\sqrt{2}} \\
\frac{2}{\pi} \ln \left(\frac{2+2 \sqrt{k}}{1-\sqrt{k}}\right) & \frac{1}{\sqrt{2}} \leq k \leq 1
\end{array} .\right.
$$

Therefore, the p.u.l. distributed capacitance $C_{E}$ can be calculated as,

$$
C_{E}=\left(\frac{1}{C_{e q}}+\frac{1}{C_{e l}}\right)^{-1}
$$


The distributed inductance $L_{E}$ of MLGNR interconnects consists of the equivalent kinetic inductance $L_{e q}$ and magnetic inductance $L_{m a}$, and their relationship can be described as in Equation (12). Similarly, the p.u.l. equivalent kinetic inductance $L_{e q}$ also can be computed by using a recursive method as [1,17],

$$
\begin{gathered}
L_{E}=L_{e q}+L_{m a}=L_{e q}+\frac{\mu_{0} T_{o x}}{W}, \\
L_{r e c}^{1}=L_{k}=h / 4 e^{2} v_{f} N_{c h} \\
L_{r e c}^{i}=\left(\frac{1}{L_{r e c}^{i-1}+L_{m}}+\frac{1}{L_{k}}\right)^{-1}, \\
L_{e q}=L_{r e c}^{N_{\text {layer }}} .
\end{gathered}
$$

Herein, $L_{k}$ represents the p.u.l. kinetic inductance of monolayer GNR. $L_{m}$ denotes the p.u.l. coupling inductance between successive GNR layers and can be expressed as $L_{m}=\mu_{0} \delta / W$ (here $\mu_{0}=$ $8.854 \times 10^{-12}$ is the vacuum magnetic permeability).

As illustrated in Figure 2, the impacts of mutual inductance $M_{m}$ and coupling capacitance $C_{c}$ on the dynamic and functional crosstalk of the coupled MLGNR interconnects are taken into consideration. The analytical expressions of p.u.l. $M_{m}$ and $C_{c}$ are defined as follows [13],

$$
\begin{gathered}
M_{m}=\frac{\mu_{0}}{2 \pi} \ln \left(\frac{2}{S+W}-1\right), \\
C_{C}=\frac{0.5}{1+\left(\frac{S}{\left(T_{g n r}+T_{o x}\right)}\right)^{2}} C_{[B C P]}\left(\frac{T_{g n r}}{S / 2}, \frac{2 T_{o x}}{S / 2}\right)+\frac{0.87}{1+\left(\frac{S / 2}{\left(T_{g n r}+T_{o x}\right)}\right)^{2}} C_{[C P]}\left(\frac{W}{S}\right) .
\end{gathered}
$$

Herein $C_{[B C P]}(z, y)$ and $C_{[C P]}(z)$ are written as below,

$$
\begin{gathered}
C_{[B C P]}(z, y)=\frac{\varepsilon_{r} \varepsilon_{0}}{2} M\left[K_{[B C P]}(z, y)\right], \\
C_{[C P]}(z)=\frac{\varepsilon_{r} \varepsilon_{0}}{4} M\left[K_{[C P]}(z)\right],
\end{gathered}
$$

wherein the function $M[$.$] is shown in Equation (10), K_{[B C P]}(\mathrm{z}, \mathrm{y})$ and $K_{[C P]}(\mathrm{z})$ are given in reference [30].

\section{Crosstalk Delay Model}

Based on the single-line delay model [31], we derived a 50\% crosstalk delay model of the coupled MLGNR interconnects considering the in-phase crosstalk and out-of-phase crosstalk as,

$$
T_{\text {delay }}=\left(1.48 \xi+e^{-2.9 \xi^{1.35}}\right) \sqrt{L_{T} L_{g n r}\left(C_{T} L_{g n r}+C_{l}\right)}
$$

Here, the total equivalent capacitance $C_{T}=C_{E}+(1-\beta) C_{c}$, the total inductance $L_{T}=L_{E}+\beta M_{m}$ and $\xi=\frac{1}{2}\left(1+\frac{C_{l}}{C_{T} L_{g n r}}\right)^{-0.5}\left[\begin{array}{l}\left(\frac{1}{2} R_{E} L_{g n r}+2 R_{l u}+R_{d}\right) \sqrt{C_{T} / L_{T}}+ \\ \left(R_{E} L_{g n r}+2 R_{l u}+R_{d}\right) \sqrt{C_{l}^{2} /\left[L_{T} \times C_{T} \times L_{g n r}{ }^{2}\right]}\end{array}\right]$.

The dynamic crosstalk consists of in-phase crosstalk and out-of-phase crosstalk schemes. For the switching factor $\beta, \beta=1$ and $\beta=-1$ are introduced to distinguish the corresponding schemes, namely, the aggressor and victim lines switching in the same direction and opposite direction, respectively. 


\section{Crosstalk Noise Model}

The ABCD parameter matrix for the MLGNR victim interconnect excluding the driver and load terminals under in-phase crosstalk and out-of-phase crosstalk schemes, respectively, can be expressed as,

$$
\begin{aligned}
& {\left[\begin{array}{cc}
A_{\text {in }} & B_{\text {in }} \\
C_{\text {in }} & D_{\text {in }}
\end{array}\right]=\left[\begin{array}{cc}
\cosh \left(\theta_{\text {in }} L_{\text {gnr }}\right) & Z_{\text {in }} \sinh \left(\theta_{\text {in }} L_{\text {gnr }}\right) \\
\frac{\sinh \left(\theta_{\text {in }} L_{\text {gnr }}\right)}{Z_{\text {in }}} & \cosh \left(\theta_{\text {in }} L_{\text {gnr }}\right)
\end{array}\right],} \\
& {\left[\begin{array}{ll}
A_{\text {out }} & B_{\text {out }} \\
C_{\text {out }} & D_{\text {out }}
\end{array}\right]=\left[\begin{array}{cc}
\cosh \left(\theta_{\text {out }} L_{\text {onr }}\right) & Z_{\text {out }} \sinh \left(\theta_{\text {out }} L_{\text {gnr }}\right) \\
\frac{\sinh \left(\theta_{\text {out }} L_{\text {gnr }}\right)}{Z_{\text {out }}} & \cosh \left(\theta_{\text {out }} L_{\text {gnr }}\right)
\end{array}\right],}
\end{aligned}
$$

where $\theta_{\text {in }}$ and $\theta_{\text {out }}$ are propagation constant of the MLGNR victim interconnect under in-phase crosstalk and out-of-phase crosstalk models, respectively. Similarly, $Z_{\text {in }}$ and $Z_{\text {out }}$ represent the corresponding characteristic impedance, respectively. They are given by,

$$
\begin{gathered}
\theta_{(\text {in }, \text { out })}=\sqrt{\left[R_{E}+s\left(L_{E}+\beta M_{m}\right)\right]\left[s\left(C_{E}+(1-\beta) C_{c}\right)\right]}, \\
Z_{(\text {in,out })}=\sqrt{\frac{R_{E}+s\left(L_{E}+\beta M_{m}\right)}{s\left(C_{E}+(1-\beta) C_{c}\right)}} .
\end{gathered}
$$

Herein $\theta_{(i n, o u t)}$ is composed of $\theta_{\text {in }}$ and $\theta_{\text {out }}$ cases, similarly, $Z_{\text {in }}$ and $Z_{\text {out }}$ are expressed as $Z_{(i n, o u t)}$. $\beta$ $=1$ is for the in-phase crosstalk scheme while $\beta=-1$ represents the out-of-phase crosstalk case.

Taking the effect of the driver terminals into account, the total ABCD parameter matrix of the MLGNR victim interconnect at different crosstalk models can be written by,

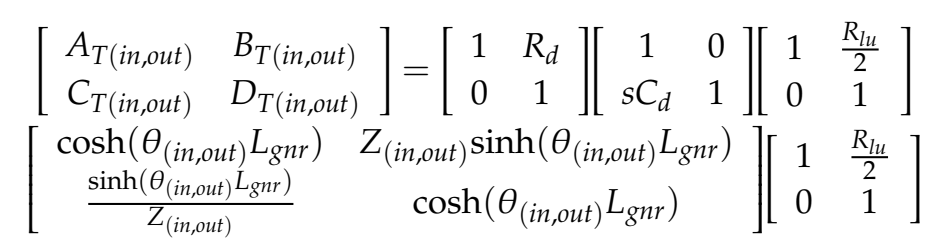

Being similar to the situation mentioned above $\left(\theta_{(i n, o u t)}\right.$ and $\left.Z_{(i n, o u t)}\right), A_{T(i n, o u t)}$ consists of $A_{T(i n)}$ and $A_{T(o u t)}$ cases, which represents the parameters of the total ABCD matrix under in-phase crosstalk and out-of-phase crosstalk models. The meaning of parameters $B_{T(i n, o u t)}, C_{T(i n, o u t)}$, and $D_{T(i n, o u t)}$ are the same as the $A_{T(\text { in,out })}$ that contain two cases of in-phase crosstalk and out-of-phase crosstalk. They can be solved by matrix computation as follows,

$$
\begin{aligned}
& A_{T(\text { in }, \text { out })}=\frac{\left[\frac{1}{2}\left(s C_{d} R_{d}+1\right) R_{l u}+R_{d}\right] \sinh \left(\theta_{(\text {in out })} L_{g n r}\right)}{Z_{(\text {in,out })}}, \\
& +\left(s C_{d} R_{d}+1\right) \cosh \left(\theta_{(i n, o u t)} L_{g n r}\right)
\end{aligned}
$$

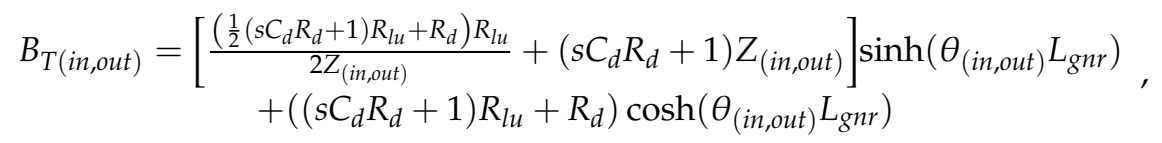

$$
\begin{aligned}
& C_{T(\text { in }, \text { out })}=\frac{\left(\frac{1}{2} s C_{d} R_{l u}+1\right) \sinh \left(\theta_{(\text {in,out })} L_{\text {gnr }}\right)}{Z_{(\text {in,out })}}+s C_{d} \cosh \left(\theta_{(\text {in }, \text { out })} L_{\text {gnr }}\right), \\
& D_{T(\text { in }, \text { out })}=\left[\frac{\left(\frac{1}{2} s C_{d} R_{l u}+1\right) R_{l u}}{2 Z_{(i n, o u t)}}+s C_{d} Z_{(\text {in }, \text { out })}\right] \sinh \left(\theta_{(\text {in }, \text { out })} L_{\text {gnr }}\right) . \\
& +\left(s C_{d} R_{l u}+1\right) \cosh \left(\theta_{(i n, o u t)} L_{g n r}\right)
\end{aligned}
$$


Combining the total $\mathrm{ABCD}$ parameter matrix described in Equation (25), the relationship between the voltage and current of input-output ports for the MLGNR victim interconnect depicted in Figure 2, can be deduced as,

$$
\left[\begin{array}{c}
V_{v i}(s) \\
I_{v i}(s)
\end{array}\right]=\left[\begin{array}{ll}
A_{T(\text { in }, \text { out })} & B_{T(\text { in,out })} \\
C_{T(\text { in,out })} & D_{T(\text { in,out })}
\end{array}\right]\left[\begin{array}{c}
V_{v o}(s) \\
I_{v o}(s)
\end{array}\right]
$$

Substituting the expression of load capacitance $I_{v o}={ }_{s} C_{l} V_{v o}$ shown in Figure 2 into Equation (26), the transfer functions of the decoupled MLGNR victim interconnect under different phase modes are derived as,

$$
\begin{gathered}
H(s)_{\text {in }}=\frac{1}{A_{T(\text { in })}+s C_{l} B_{T(\text { in })}}=\left(1+\sum_{i=1}^{5}\left(a_{i} s^{i}\right)\right)^{-1}, \\
H(s)_{\text {out }}=\frac{1}{A_{T(\text { out })}+s C_{l} B_{T(\text { out })}}=\left(1+\sum_{i=1}^{5}\left(b_{i} s^{i}\right)\right)^{-1} .
\end{gathered}
$$

Herein, in order to ensure the signal integrity characteristics at the output port of MLGNR victim interconnect, the transfer functions are approximated by adopting a fifth-order pade's expansion.

The crosstalk noise is usually defined as the functional crosstalk. It can be defined that the aggressor line switches from logic 0 to logic 1 while the victim line keeps in a quiescent state of logic 0 . Based on the principle of the functional crosstalk, a peak voltage will be observed at output port of the victim line when the aggressor line switches owing to the coupled crosstalk. The noise output of the MLGNR victim interconnect induced by the switching of aggressor line can be obtained as follows [32],

$$
V_{\text {noise }}(s)=\frac{1}{2} V_{\text {agg }}(s)\left(H(s)_{\text {in }}-H(s)_{\text {out }}\right),
$$

where $V_{\text {noise }}(s)$ represents the noise output signal of the MLGNR victim interconnect in the Laplace domain. Here the input port of MLGNR aggressor line is defined into an ideal step-response signal $V_{\text {agg }}(s)=1 / \mathrm{s}$. We can obtain the noise output signal in the time domain by applying the inverse Laplace transform for the Equation (29) as,

$$
V_{\text {noise }}(t)=L^{-1}\left[\frac{1}{2} V_{\text {agg }}(s)\left(H(s)_{\text {in }}-H(s)_{\text {out }}\right)\right] .
$$

\section{Results and Discussions}

This section investigates the impacts of different dielectric materials on crosstalk delay, transfer gain, and noise output signal of coupled MLGNR interconnect at global level of $7 \mathrm{~nm}$ technology node. All geometrical and physical electrical parameters were extracted from references [33,34] as follows, $W$ $=11.5 \mathrm{~nm}, T_{\text {gnr }}=26.91 \mathrm{~nm}, S=11.5 \mathrm{~nm}, T_{o x}=17.25 \mathrm{~nm}, E_{f}=0.3 \mathrm{eV}, \varepsilon_{0}=1.95 \times 10^{-11} \mathrm{~F} / \mathrm{m}, \mu_{0}=4 \pi \times$ $10^{-7}, P=0, R_{d}=20.51 \mathrm{k} \Omega, C_{d}=0.063 \mathrm{fF}, C_{l}=0.2 \mathrm{fF}$. Herein, $R_{d}, C_{d}$, and $C_{l}$ are the equivalent values of minimum-sized gate. In general, the sizes of driver and load are 100 times larger than that of the minimum-sized gate at global level $\left(100 \mu \mathrm{m} \leq L_{g n r} \leq 10 \mathrm{~mm}\right)$ interconnects [35], then their values can be rewritten as, $R_{d}{ }^{\prime}=R_{d} / 100, C_{d}{ }^{\prime}=C_{d} \times 100$ and $C_{l}{ }^{\prime}=C_{l} \times 100$. All the numerical simulation results presented in the next section were obtained by carrying out the MATLAB R2013a.

In order to compare the impacts of different dielectric mediums on delay time of coupled MLGNR interconnect, the crosstalk delay of victim line versus interconnect length under different phase modes were obtained by the Equation (20), as displayed in Figure 3. 


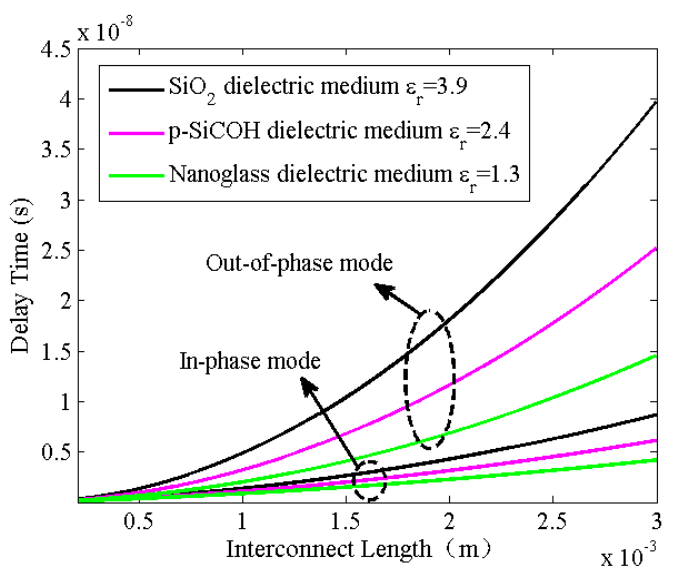

Figure 3. The impacts of different dielectric materials on crosstalk delay of victim line versus interconnect length under in-phase and out-of-phase modes.

As shown in Figure 3, it can be observed that the crosstalk delay for the coupled MLGNR interconnect with the nanoglass $\left(\varepsilon_{r}=1.3\right)$ as the dielectric medium is less than that of the conventional $\mathrm{SiO}_{2}$ dielectric medium under in-phase and out-of-phase modes. Taking the interconnect length of $L_{g n r}=2000 \mu \mathrm{m}$ as an instance, the delay time under out-of-phase mode for the nanoglass medium is $6.886 \mathrm{~ns}$ while for the $\mathrm{SiO}_{2}$ dielectric medium is $18.115 \mathrm{~ns}$. Similarly, the delay time at in-phase mode for nanoglass and $\mathrm{SiO}_{2}$ dielectric mediums are $2.277 \mathrm{~ns}$ and $4.286 \mathrm{~ns}$ in the same length as the former, respectively. The reason behind this is that the electrostatic capacitance and coupling capacitance will reduce as the relative dielectric constant $\varepsilon_{r}$ decrease, thereby, leading to a lesser total equivalent capacitance $C_{T}$. In combination with Equation (20), it is evident that the crosstalk delay is approximately in positive proportion with the total equivalent capacitance $C_{T}$. In addition, according to our numerical simulation results, the maximum difference of delay time between $\mathrm{SiO}_{2}$ and nanoglass dielectric mediums can reach to $25.202 \mathrm{~ns}$ for an interconnect length of $L_{g n r}=3000 \mu \mathrm{m}$ at the out-of phase crosstalk. Thus, replacing the traditional $\mathrm{SiO}_{2}$ with the ultra-low-k dielectric material is an efficient way to reduce crosstalk delay of coupled interconnects.

Moreover, it is clearly shown from Figure 3 that the crosstalk delay of coupled MLGNR interconnects at out-of-phase mode is significantly higher than that of in-phase crosstalk mode for all dielectric materials. Giving the interconnect length of $L_{g n r}=2500 \mu \mathrm{m}$ as an example, the delay time for $\mathrm{SiO}_{2}$ dielectric medium under in-phase and out-of-phase modes are $6.308 \mathrm{~ns}$ and $27.883 \mathrm{~ns}$. The corresponding values for $\mathrm{p}-\mathrm{SiCOH}$ dielectric medium are $4.525 \mathrm{~ns}$ and $17.802 \mathrm{~ns}$, and the case for nanoglass dielectric medium are $3.173 \mathrm{~ns}$ and $10.364 \mathrm{~ns}$. This can be explained by the Miller coupling capacitance, which only exists in out-of-phase crosstalk mode, causing the total equivalent capacitance of the MLGNR victim interconnects under out-of-phase mode to be greater than that of in-phase mode.

Figure 4 shows the frequency response of MLGNR victim line with the interconnect length $L_{g n r}$ $=1000 \mu \mathrm{m}$ under in-phase and out-of-phase modes for different dielectric materials. Transfer gain represents the magnitude of frequency response of the interconnect system and is the ratio of amplitude between the output and input signal at different frequencies. The transfer gain under in-phase and out-of-phase can be obtained by the Equations (27) and (28), respectively. As shown in Figure 4, in the high frequency region, it is obvious that the transfer gain increases as the relative dielectric constant $\varepsilon_{r}$ decreases for in-phase and out-of-phase modes. This is due to the fact that the coupled MLGNR interconnects system can be considered as the RC low pass filter and its cut-off frequency is approximately expressed as: $1 /\left(2 \pi \times C_{T} \times R_{E}\right)$ [16], and the total capacitance $C_{T}$ of the victim line decreases with the decrease of $\varepsilon_{r}$. Thus the MLGNR victim interconnect for using the nanoglass has a larger cut-off frequency compared with the $\mathrm{p}-\mathrm{SiCOH}$ and $\mathrm{SiO}_{2}$ cases. 


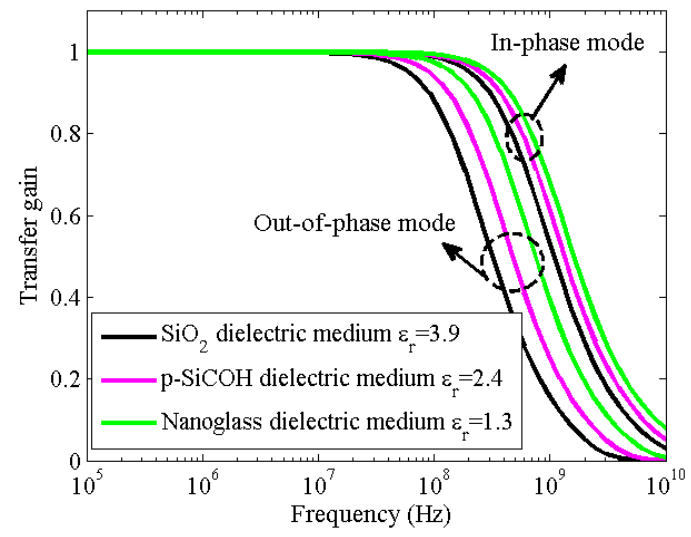

Figure 4. The impacts of different dielectric materials on frequency response of victim line under in-phase and out-of-phase modes. Here, the interconnect length $L_{g n r}=1000 \mu \mathrm{m}$.

Moreover, it can be found from Figure 4 that transfer gain of coupled MLGNR interconnects under in-phase mode is evidently greater than that of out-of-phase crosstalk mode for any dielectric materials. The reason for this phenomenon is that the total capacitance of the victim MLGNR interconnect under out-of-phase mode is larger than that of in-phase mode. Therefore, the former will have a lesser cut-off frequency compared with the latter.

Based on the Equation (30), the effect of different dielectric mediums on crosstalk noise of victim MLGNR interconnect is illustrated in Figure $5 \mathrm{a}$, meanwhile the peak noise voltage regarding the indispensable noise parameter for different dielectric mediums versus interconnect length is described in Figure 5b.

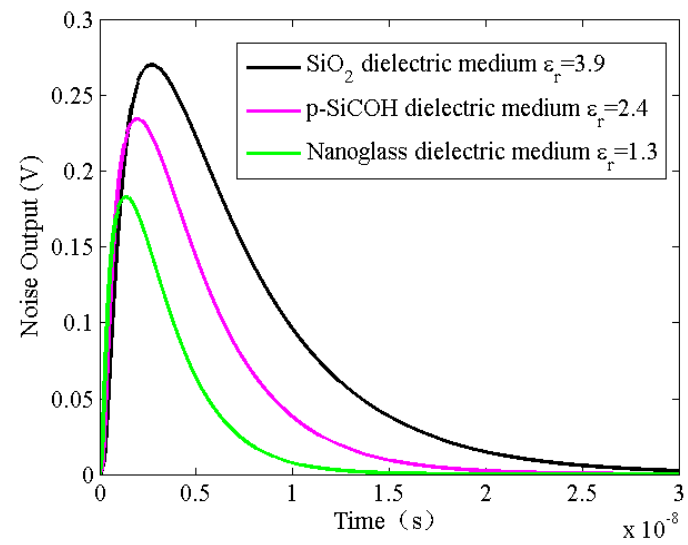

(a)

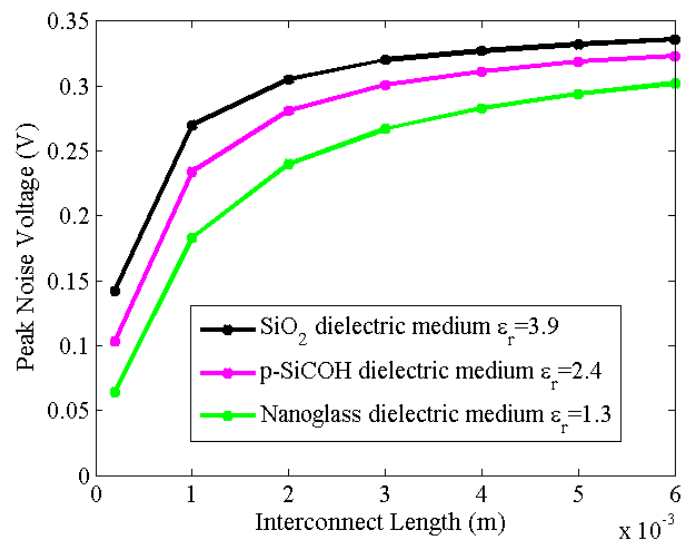

(b)

Figure 5. The impacts of different dielectric materials on (a) noise output voltage with interconnect length $L_{g n r}=1000 \mu \mathrm{m}$ and (b) peak noise voltage for different interconnect length.

It is remarkable from Figure 5a that the peak noise voltage will decrease with the decrease of the relative dielectric constant $\varepsilon_{r}$. For instance, the peak noise voltage with the interconnect length $L_{g n r}=1000 \mu \mathrm{m}$ for $\mathrm{SiO}_{2}, \mathrm{p}$-SiCOH and nanoglass dielectric mediums are $0.270 \mathrm{~V}, 0.234 \mathrm{~V}$, and $0.183 \mathrm{~V}$, respectively. It can be explained that the crosstalk noise is induced by the coupling capacitance $C_{c}$ and mutual inductance $M_{m}$ existing on the position between aggressor and victim MLGNR interconnect, as shown in Figure 2. On the other hand, it is noteworthy that the Miller coupling capacitance is the dominant factor of forming the crosstalk noise. Certainly, there is no doubt according to the Equations (17)-(19) that a lesser relative dielectric constant $\varepsilon_{r}$ can result in a smaller Miller coupling capacitance.

Moreover, as shown in Figure $5 b$, it is obviously found that the peak noise voltage of coupled MLGNR interconnect increases as the interconnect length increases for all different dielectric mediums. This is due to the fact that the increases of interconnect length give rise to Miller coupling capacitance. 
In addition, it is shown from Figure $5 b$ that applying the ultra-low-k dielectric material to reduce the peak noise voltage is very obvious at global level interconnects. Based on the numerical simulation results, the maximum difference of peak noise voltage between the $\mathrm{SiO}_{2}$ and nanoglass dielectric mediums can reach to $0.102 \mathrm{~V}$ when the interconnect length $L_{g n r}=1000 \mu \mathrm{m}$, and the corresponding value for the minimum difference is $0.038 \mathrm{~V}$ when the interconnect length is chosen as $L_{g n r}=6000 \mu \mathrm{m}$.

\section{Conclusions}

Based on the transmission line model, an equivalent distributed circuit of coupled MLGNR interconnects was established and the coupling capacitance and mutual inductance were taken into consideration. By using the extracted parameters, the impacts of different dielectric materials on crosstalk delay, noise output voltage, and transfer gain were predicted. The numerical simulation results showed that substituting the conventional $\mathrm{SiO}_{2}$ dielectric medium with the ultra-low-k dielectric material for the coupled MLGNR interconnects has a greater performance advantage in terms of the crosstalk delay, noise output, and transfer gain at the same conditions. Furthermore, it was found that the coupled MLGNR interconnect under out-of-phase mode has a greater crosstalk delay and a lesser transfer gain compared with under in-phase mode for all dielectric material, and the peak noise voltage of coupled MLGNR interconnect increases as the interconnect length increases. In the light of our simulation results, it can be expected that the ultra-low-k dielectric materials may be an emerging technology to improve the performance of crosstalk delay, noise output and transfer gain of the coupled MLGNR interconnects.

Author Contributions: Conceptualization, P.X.; methodology, P.X. and Z.P.; formal analysis, P.X. and Z.P.; modeling, P.X. and Z.P.; validation, P.X. and Z.P.; data curation, P.X. and Z.P.; writing一original draft preparation, P.X.; writing-review and editing, P.X., Z.P. and Z.T.

Funding: This work was supported by Guangzhou Science and Technology Project (grant No. 201904010107), Guangdong Province Science and Technology Project (grant No. 2016B090918071), and National Natural Science Foundation of China (grant No. 61072028).

Acknowledgments: The authors would like to thank editors and anonymous reviewers for their special effort.

Conflicts of Interest: The authors declare no conflict of interest.

\section{References}

1. Zhao, W.S.; Yin, W.Y. Comparative study on multilayer graphene nanoribbon (MLGNR) interconnects. IEEE Trans. Electromagn Compat. 2014, 56, 638-645. [CrossRef]

2. Liang, F.; Wang, G.; Lin, H. Modeling of crosstalk effects in multiwall carbon nanotube interconnects. IEEE Trans. Electromagn. Compat. 2012, 54, 133-139. [CrossRef]

3. Bagheri, A.; Ranjbar, M.; Haji-Nasiri, S.; Mirzakuchaki, S. Crosstalk bandwidth and stability analysis in graphene nanoribbon interconnects. Microelectron. Reliab. 2015, 55, 1262-1268. [CrossRef]

4. Rai, M.K.; Garg, H.; Kaushik, B.K. Temperature-Dependent Modeling and Crosstalk Analysis in Mixed Carbon Nanotube Bundle Interconnects. J. Electron. Mater. 2017, 46, 5324-5337. [CrossRef]

5. Bagheril, A.; Ranjbar, M.; Haji-Nasiri, S.; Mirzakuchaki, S. Modelling and analysis of crosstalk induced noise effects in bundle SWCNT interconnects and its impact on signal stability. J. Comput. Electron. 2017, 16, 845-855. [CrossRef]

6. Gengchiau, L.; Neophytos, N.; Nikonov, D.E.; Lundstrom, M.S. Performance projections for ballistic graphene nano-ribbon field-effect transistor. IEEE Trans. Electron Devices 2007, 54, 677-682.

7. Kaur, T.; Rai, M.K.; Khanna, R. Effect of temperature on the performance analysis of MLGNR interconnects. J. Comput. Electron. 2019, 18, 722-736. [CrossRef]

8. Kanthamani, S.; Gayathiri, G.; Rohini, S. Meshless analysis of bilayer graphene nanoribbon for radio frequency interconnects. Micro. Nano Lett. 2015, 10, 613-616. [CrossRef]

9. Kumar, V.R.; Majumder, M.K.; Kaushik, B.K. Graphene based on-chip interconnects and TSVs: Prospects and challenges. IEEE Nanotechnol. Mag. 2014, 8, 14-20. [CrossRef] 
10. Kumar, V.; Rakheja, S.; Naeemi, A. Performance and energy-per-Bit modeling of multilayer graphene nanoribbon conductors. IEEE Trans. Electron. Devices 2012, 59, 2753-2761. [CrossRef]

11. Bhattacharya, S.; Das, S.; Mukhopadhyay, A.; Das, D. Analysis of a temperature-dependent delay optimization model for GNR interconnects using a wire sizing method. J. Comput. Electron. 2018, 17, 1536-1548. [CrossRef]

12. Nishad, A.K.; Sharma, R. Lithium-intercalated graphene interconnects: Prospects for on-Chip applications. IEEE J. Electron. Devices 2016, 4, 485-489. [CrossRef]

13. Agrawal, Y.; Kumar, M.G.; Chandel, R. A novel unified model for copper and MLGNR interconnects using voltage-and current-mode signaling schemes. IEEE Trans. Electromagn. Compat. 2017, 59, 217-227. [CrossRef]

14. Das, S.; Das, D.; Rahaman, H. Electro-thermal RF modeling and performance analysis of graphene nanoribbon interconnects. J. Comput. Electron. 2018, 17, 1695-1708. [CrossRef]

15. Kumar, V.R.; Majumder, M.K.; Alam, A. Stability and delay analysis of multi-layered GNR and multi-walled CNT interconnects. J. Comput. Electron. 2015, 14, 611-618. [CrossRef]

16. Kumar, V.R.; Majumder, M.K.; Kukkam, N.R.; Kaushik, B.K. Time and frequency domain analysis of MLGNR interconnects. IEEE Trans. Nanotechnol. 2015, 14, 484-492. [CrossRef]

17. Sahoo, M.; Rahaman, H. Modeling and analysis of crosstalk induced overshoot/undershoot effects in multilayer graphene nanoribbon interconnects and its impact on gate oxide reliability. Microelectron. Reliab. 2016, 63, 231-238. [CrossRef]

18. Morgen, M.; Ryan, E.T.; Zhao, J.; Hu, C. Low dielectric constant materials for ULSI interconnects. Annu. Rev. Mater. Res. 2000, 30, 645-680. [CrossRef]

19. Shamiryan, D.; Abell, T.; Iacopi, F. Low-k dielectric materials. Mater. Today 2004, 7, 34-39. [CrossRef]

20. Grill, A.; Gates, S.M.; Ryan, T.E.; Nguyen, S.; Priyadarshini, D. Progress in the development and understanding of advanced low $\mathrm{k}$ and ultralow $\mathrm{k}$ dielectrics for very large-scale integrated interconnects-State of the art. Appl. Phys. Rev. 2014, 1, 011306. [CrossRef]

21. Volksen, W.; Miller, R.D.; Dubois, G. Low dielectric constant materials. Chem. Rev. 2010, 110, 56-110. [CrossRef] [PubMed]

22. Si, J.J.; Ono, H.; Uchida, K. Correlation between the dielectric constant and porosity of nanoporous silica thin films deposited by the gas evaporation technique. Appl. Phys. Lett. 2001, 79, 3140-3142. [CrossRef]

23. Nishad, A.K.; Sharma, R. Performance improvement in SC-MLGNRs interconnects using interlayer dielectric insertion. IEEE Trans. Emerg. Top. Comput. 2015, 3, 470-482. [CrossRef]

24. Rai1, M.K.; Arora1, S.; Kaushik, B.K. Temperature-dependent modeling and performance analysis of coupled MLGNR interconnects. Int. J. Circ. Theory Appl. 2017, 46, 299-312. [CrossRef]

25. Nasiri, S.H. Compact formulae for number of conduction channels in vatious types of graphene nanoribbons at various temperatures. Mod. Phys. Lett. B 2012, 26, 1150004. [CrossRef]

26. Majumder, M.K.; Kukkam, N.R.; Kaushik, B.K. Frequency response and bandwidth analysis of multi-layer graphene nanoribbon and multi-walled carbon nanotube interconnects. Micro. Nano Lett. 2014, 9, 557-560. [CrossRef]

27. Gunlycke, D.; Lawler, H.M.; White, C.T. Room temperature ballistic transport in narrow graphene strips. Phys. Rev. B 2007, 75, 085418. [CrossRef]

28. Hazra, A.; Basu, S. Graphene nanoribbon as potential on-chip interconnect material-A review. C 2018, 4, 49. [CrossRef]

29. Rai1, M.K.; Chatterjee, A.K.; Sarkar, S.; Kaushik, B.K. Performance analysis of multilayer graphene nanoribbon (MLGNR) interconnects. J. Comput. Electron. 2016, 15, 358-366. [CrossRef]

30. Stellari, F.; Lacaita, A.L. New formulas of interconnect capacitances based on results of conformal mapping method. IEEE Trans. Electron Devices 2000, 47, 222-231. [CrossRef]

31. Amore, M.D.; Sarto, M.S.; Tamburrano, A. Fast transient analysis of next generation interconnects based on carbon nanotubes. IEEE Trans. Electromagn. Compat. 2010, 52, 496-503. [CrossRef]

32. Zhang, J.; Friedman, E.G. Decoupling technique and crosstalk analysis for coupled RLC interconnects. In Proceedings of the 2004 IEEE International Symposium on Circuits and Systems, Vancouver, BC, Canada, 23-26 May 2004; pp. 521-524.

33. International Technology Roadmap for Semiconductors (ITRS). Available online: http://www.itrs2.net/ (accessed on 10 July 2000). 
34. Zhao, W.; Cheng, Z.; Wang, J.; Fu, K.; Wang, D.; Zhao, P.; Wang, G.; Dong, L. Vertical graphene nanoribbon interconnects at the end of the roadmap. IEEE Trans. Electron Devices 2018, 65, 2632-2637. [CrossRef]

35. Xu, P.; Pan, Z. The analytical model for crosstalk noise of current-mode signaling in coupled RLC interconnects of VLSI circuits. J. Semicond. 2017, 38, 095003. [CrossRef] 